\title{
Recettes Alimentaires A Base Des Graines De Boscia Senegalensis Au Niger : Cas Des Communes De Bambeye Et Banibangou
}

\author{
Haoua Sabo \\ Maman Nafiou Aminou Illia \\ Maman Moustapha Rabiou
}

Département de chimie, Faculté des sciences et Technique,

Université Abdou Moumouni de Niamey Niger

\section{Aminatou Bako Maazou}

Département des Sciences Biologiques Appliquées,

Faculté des Sciences de la Santé, Université Abdou Moumouni de Niamey

\section{Soumana Douma}

Département de Biologie, Faculté des sciences et Technique,

Université Abdou Moumouni de Niamey

\section{Issa Chaibou}

Département des Sciences et Techniques de l'Elevage, Faculté d'Agronomie et des Sciences de 1'Environnement, Université Dan Dicko Dan Koulodo de Maradi

\section{Ibrahim Amoukou}

Département de productions végétales, Faculté d'Agronomie, Université Abdou Moumouni de Niamey

\section{Hamidou Idrissa ONG CONCERN}

Doi: 10.19044/esj.2018.v14n36p195 URL:http://dx.doi.org/10.19044/esj.2018.v14n36p195

\begin{abstract}
Food insecurity is an almost permanent reality in Niger due to climatic hazards. This study is a contribution to the capitalization and enhancement of Community know-how in the exploitation of natural resources and addresses the role of spontaneous foods of plant origin in the search for diversity and dietary balance, specifically the potentialities of Boscia senegalensis. It is a species whose different parts are used for many purposes in Niger. The different recipes based on immature seeds processed in two departments of Niger, Banibangou and Bambèye are listed. To this end, a questionnaire was sent to 128 women in Bambèye and 114 in Banibangou. The recipes identified are those implemented by women from these different communities. A total
\end{abstract}


of 7 recipes as well as their ingredients and methods of preparation have been identified. One is made from seeds only. Three (3) recipes are prepared in association with millet, cowpea, Gynandropsis gynandra leaves and Maerua crassifola. For two (2) recipes, the seeds are taken back with milk. Finally, one (1) recipe using Boscia senegalensis bark and Guéra senegalensis leaves is used in postpartum women. In view of these results, it would be important to create the conditions for maintaining them, for their sustainable use, for their inclusion in agricultural and food statistics and for the dissemination of these revenues in other regions.

Keywords: Seeds, Boscia senegalensis, recipes, Balance, diversification, food

\section{Résumé}

L'insécurité alimentaire est une réalité presque permanente au Niger du fait des aléas climatiques. La présente étude est une contribution à la capitalisation et la valorisation du savoir-faire communautaire dans l'exploitation des ressources naturelles et aborde la place des aliments spontanés d'origine végétale dans la recherche de diversité et l'équilibre alimentaire, de manière spécifique, les potentialités de Boscia senegalensis. Il s'agit d'une espèce dont les différentes parties servent des nombreux usages au Niger. Les différentes recettes à base des graines immatures traitées dans deux départements du Niger, Banibangou et Bambèye sont répertoriées. A cet effet, un questionnaire a été adressé à 128 femmes à Bambèye et 114 à Banibangou. Les recettes identifiées sont celles mises en œuvre par les femmes de ces différentes communautés. Au total 7 recettes ainsi que leurs ingrédients et modes de préparations ont été recensés. Une est confectionnée à base des graines uniquement. Trois (3) recettes sont préparées en association avec le mil, le niébé, les feuilles de Gynandropsis gynandra et Maerua crassifola. Pour deux (2) recettes, les graines sont reprises avec du lait. Enfin, une (1) recette faisant-intervenir les écorces de Boscia senegalensis et des feuilles de Guéra senegalensis est utilisée chez les femmes en post-partum. Au vue de ces résultats il serait important de créer les conditions de maintien, de leur exploitation durable, de leurs prises en compte dans les statistiques agricoles et alimentaires et la vulgarisation de ces recettes dans les autres régions.

Mots clés: Graines, Boscia senegalensis, recettes, Equilibre, diversification, alimentaire 


\section{Introduction}

Au Niger, les céréales traditionnelles, le mil et le sorgho, constituent la base du régime alimentaire des Nigériens et apportent l'essentiel de l'énergie (près des trois quarts) (INS, 2016) et des protéines. Les légumineuses et/ou les oléagineux sont surtout utilisés pour la préparation des sauces. Les fruits et les légumes sont consommés exclusivement de façon saisonnière. La consommation de viande intéresse principalement les villes, et celle du poisson n'est significative qu'autour du fleuve Niger, du lac Tchad et des rivières. Le lait et le sucre sont consommés de manière variable en fonction des habitudes alimentaires, de la disponibilité de ces denrées et des revenus des consommateurs. L'huile n'est consommée en quantité importante qu'en milieu urbain (INS, 2016). Les fluctuations climatiques, notamment les sécheresses répétées, ont plongé de façon presque cyclique le Niger dans de sévères crises alimentaires (famines de 1973, 1984, 2001, 2005, 2010 et 2012) (Alpha Gado, 1989) et ont fragilisé l'environnement et les systèmes agraires (Ambouta et al, 1996). Pour survivre, les populations adoptent des stratégies qui vont des migrations à l'exploitation des maigres ressources naturelles. Le gouvernement, quant à lui, intervient à travers divers programmes de réduction de la pauvreté et de sécurité alimentaire. Dans la mise en œuvre de cette nouvelle approche des réflexions sont engagées afin de prendre en compte le savoir local.

$\mathrm{Au}$ Niger, en période de famine, les populations ont quasisystématiquement recours à des aliments spontanés de cueillette d'origine végétale (feuilles, fleurs et fruits des ligneux comme des herbacées). Théoriquement, plus la diversité alimentaire est faible, plus grand est le risque d'apports insuffisants en micronutriments et donc de carences nutritionnelles (INS, 2012). La diversité alimentaire au niveau ménage a été étudiée en 2016 dans l'enquête nationale de sécurité alimentaire. Sur la base du Score de Consommation Alimentaire (SCA) qui renseigne sur l'accès des ménages aux différents groupes d'aliments, seulement 57\% des ménages ruraux étaient classés comme ayant un score (i.e. diversité) acceptable, tandis que chez $18 \%$ la diversité était classée comme pauvre, c'est-à-dire un régime alimentaire monotone essentiellement composé de céréales et de très peu d'aliments riches en micronutriments de bonne biodisponibilité, tels que les aliments d'origine animale et les fruits. Suivant la région administrative, il ressort que les régions les plus affectées par le déséquilibre alimentaire sévère sont celles de Dosso (21\%), Tahoua (19\%), Tillabéri (19\%) et Zinder (18\%) (INS, 2016). De plus, la répartition des ménages selon les classes de score de consommation alimentaire indique que $22 \%$ des ménages vivant en milieu rural ont une alimentation pauvre ; $24 \%$ une alimentation limite et $55 \%$ une alimentation acceptable (INS, 2016). Il apparaît donc nécessaire d'initier une étude de capitalisation des connaissances paysannes sur les ressources naturelles et de 
créer les conditions de maintien et de leur exploitation durable et leurs prises en compte dans l'atteinte de l'équilibre alimentaire. C'est dans cette perspective qu'a été entrepris cette étude sur les connaissances paysannes et les usages de Boscia senegalensis afin de promouvoir des réflexes de sa protection et de sa conservation.

Boscia senegalensis est un arbuste de la famille des caparacées (capparidacées) dont l'aire de distribution s'étend du Sénégal/Mauritanie à la Somalie (Arbonnier, 2009) On la rencontre pratiquement sur l'ensemble du territoire nigérien (Saadou, 1996). Les usages que l'on fait de Boscia sont multiples et vont de l'alimentation à la protection de l'environnement en passant par la pharmacopée et la phytopharmacie. (Kari et al., 2004 ; Khadra, 2006 ; Ermias et al., 2008 ; Doka and Yagi, 2009 ; Mohamed, 2009 ; Victoria and Musa et al., 2011 ; Mirutse and Tilahun, 2013). En effet, Boscia est particulièrement connue des populations nigériennes pour sa contribution dans la gestion des crises alimentaires et pour ses vertus pharmacologiques et phytosanitaires. Lors des disettes les graines de Boscia constituent le principal aliment de subsistance des certains ménages et ceci pendant 5 à 6 mois. (Baumer, 1981 ; Becher, 1983 ; Maydell, 1983 ; Booth and Wickens, 1988 ; Salih et al., 1991; Kim et al., 1997; Arbonnier, 2009 ; Dicko et al., 2001). Travaillant sur la composition nutritive des graines de Boscia, Kim et al. (1997) ont mis en évidence l'existence de quantités importantes d'acides aminés essentiels et du fer. De leur côté ; Salih et al. (1991) ont montré que la qualité nutritive des graines est comparable à celle du sorgho, mais que le prétraitement par ébouillantage des graines réduit le taux des matières azotées.

La présente étude part de l'hypothèse qu'il existe des connaissances locales sur la technologie de transformation de ce produit qu'il faille identifier, évaluer et vulgariser les plus appropriées dans les zones où les peuplements de cette espèce sont importants. Au vue des multiples usages des graines de Boscia senegalensis, une enquête a été conduite pour répertorier les différentes recettes dans deux départements du Niger de grande consommation.

\section{Matériels et méthodes \\ Présentation de la zone d'étude}

L'étude a été conduite dans deux des huit régions que compte le Niger. Il s'agit spécifiquement de la commune de Banibangou situé dans la région de Tillabéri (15'2'27' 'N 2 ${ }^{\circ} 42^{\prime} 18^{\prime}$ ' E) et de la commune de Bambèye situé dans la région de Tahoua $\left(5^{\circ} 5^{\prime} 20^{\prime}\right.$ 'N et $14^{\circ} 42^{\prime} 58^{\prime}$ 'E). Ces deux communes sont situées en zone sahélienne où la pluviosité annuelle varie entre 250 et $300 \mathrm{~mm}$. Le choix de ces deux sites a été fait pour prendre en compte la diversité culturelle et voir les variations des recettes culinaires des graines de Boscia en fonction des coutumes Zarma et Haoussa. 


\section{Matériel végétal}

Il est constitué des graines immatures de Boscia senegalensis achetées sur le marché local. Dans la préparation des différentes recettes, des feuilles de Gynandropsis gynandra de Maerua crassifola, les graines de Vigna Unguilata (Niebé) associées sont également achetées localement ainsi que le lait.

\section{Méthode}

Un questionnaire sur les différentes recettes à base des graines de Boscia senegalensis a été administré à 128 femmes à Bambèye et 114 à Banibangou par commodité au cours d'une première mission. Après le dépouillement des données, 7 recettes sont recensées. Lors d'une deuxième mission, des femmes ont été recrutées pour assurer la démonstration culinaire. Les différents ingrédients sont achetés sur les marchés locaux.

Les graines immatures de Boscia senegalensis ont été traitées après plusieurs lavages à l'eau pour faire partir l'amertume. Les graines prétraitées ainsi que tous les autres ingrédients sont bouillies séparément.
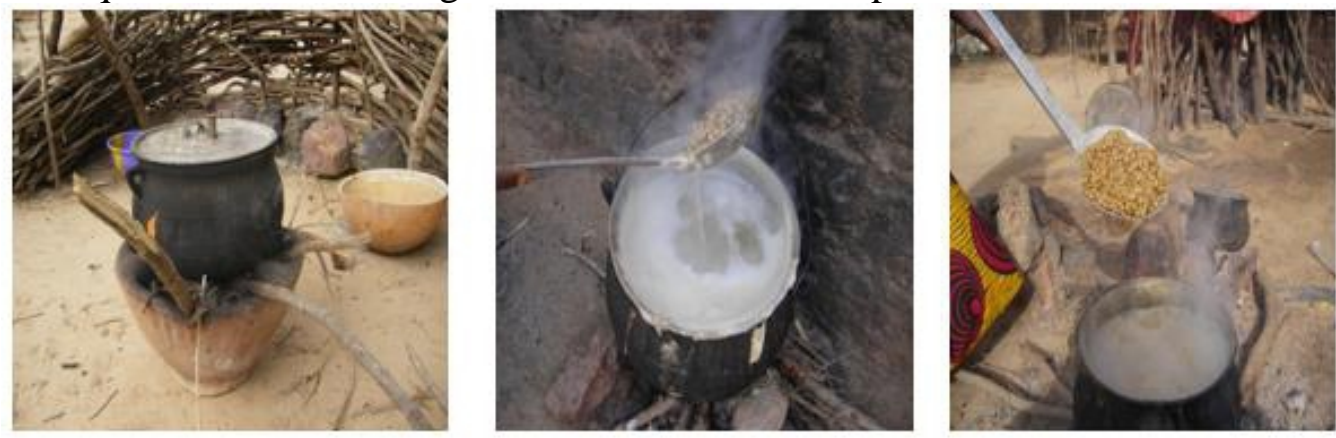

Figure 1: cuisson des graines de Boscia Senegalensis prétraitées

\section{Résultats et discussion Présentation des Résultats}

$\mathrm{Au}$ total 7 recettes ont été mises en œuvre dont 4 à Bambeye et 3 à Banibangou. Le tableau I donne le récapitulatif des différentes recettes.

Tableau I : Récapitulatif des différentes recettes obtenues à base des graines de Boscia senegalensis

\begin{tabular}{|l|l|}
\hline Recettes de Bambèye & Recettes de Banibangou \\
\hline $\begin{array}{l}\text { Graines de Boscia senegalensis (Anza, nom local } \\
\text { de l'espèce) plus Niébé (vigna unguigulata) }\end{array}$ & $\begin{array}{l}\text { Graines de Boscia senegalensis } \\
\text { plus Hassu (feuilles de Maerua } \\
\text { crassifola) }\end{array}$ \\
\hline $\begin{array}{l}\text { Boule des Graines de Boscia senegalensis plus } \\
\text { du lait }\end{array}$ & $\begin{array}{l}\text { Graine de Boscia senegalensis } \\
\text { plus du lait }\end{array}$ \\
\cline { 1 - 2 } $\begin{array}{l}\text { Graines de Boscia senegalensis plus les feuilles } \\
\text { de gashiya (Gynandropsis gynandra) }\end{array}$ & $\begin{array}{l}\text { Coucous des Graines de Boscia } \\
\text { senegalensis } *\end{array}$ \\
\cline { 1 - 1 } $\begin{array}{l}\text { Lalumé de Boscia senegalensis (Bouillie à base } \\
\text { des écorces de Boscia senegalensis) }\end{array}$ & \\
\hline
\end{tabular}


Les tableaux II à VIII présentent dans les détails, les ingrédients et les modes de préparation des différentes recettes.

Tableau II : Recette de Graines de Boscia senegalensis plus Niébé (vigna unguigulata)

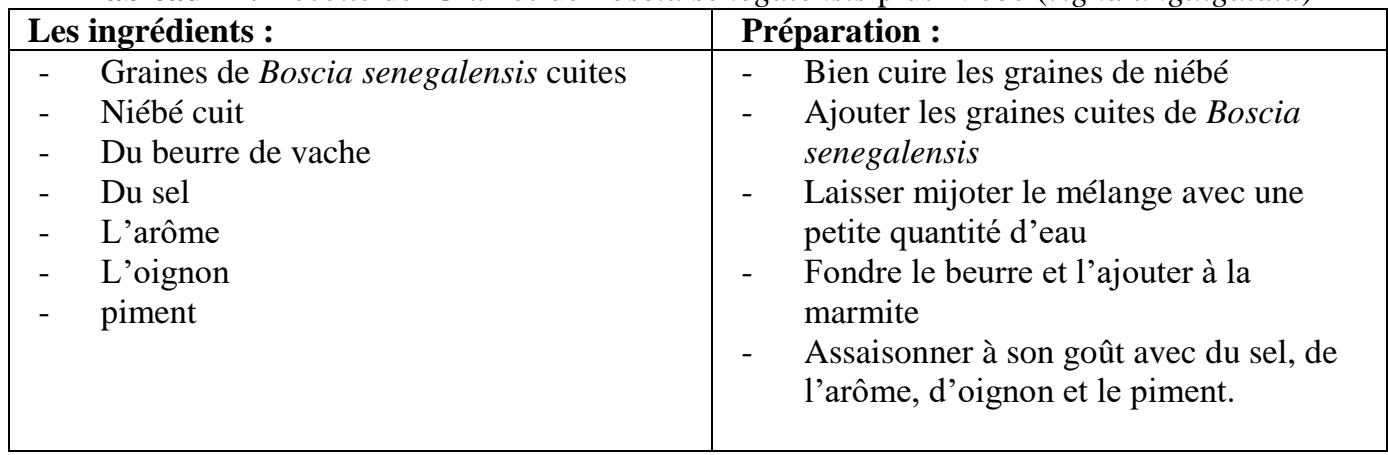

Le niébé, légumineuse riche en protéines et surtout en lysine (FAO, 2012) et qui est d'ailleurs considérée " comme la viande du pauvre » dans notre société se trouve parmi les ingrédients. On constate aisément que l'équilibre est atteint avec le beurre, lipide d'origine animal, riche en vitamines liposolubles qui est ajouté. L'oignon et les épices viennent à leur tour combler les apports en certaines vitamines et minéraux. Il ne faut pas perdre de vue la qualité nutritionnelle des graines de Boscia senegalensis, qui sont riches en glucides, minéraux et certains acides aminés.

Tableau III : Recette de Boule des Graines de Boscia senegalensis plus du lait

\begin{tabular}{|c|c|}
\hline Les ingrédients : & Préparation : \\
\hline $\begin{array}{ll}\text { - } & \text { les graines de Boscia senegalensis cuites } \\
\text { - } & \text { Boule de mil bouillies } \\
\text { - } & \text { du lait caillé } \\
\text { - } & \text { du sucre }\end{array}$ & 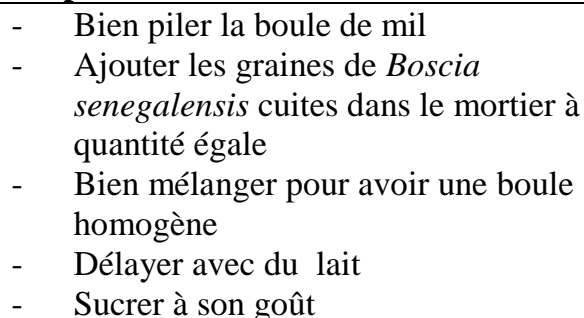 \\
\hline
\end{tabular}

Habituellement au Niger, la boule est confectionnée uniquement à base des céréales et surtout le mil (Pennisetum glaucum). La boule est bien ancrée dans les habitudes alimentaires des communautés et contribue à la diversification alimentaire surtout en milieu rural. Les graines de Boscia senegalensis, le lait et le sucre viennent renforcer la qualité nutritionnelle de cette forme de boule. 
Tableau IV : Recette de Graines de Boscia senegalensis plus les feuilles de gashiya (Gynandropsis gynandra)

\begin{tabular}{|c|c|}
\hline Les ingrédients : & Préparation : \\
\hline - $\quad$ Graines de Boscia senegalensis cuites & - $\quad$ Bien mélanger les graines de Boscia \\
\hline - $\quad$ Les feuilles cuites & senegalensis et les feuilles en les \\
\hline - L'huile & malaxant \\
\hline - Des tourteaux d'arachide & - Puis assaisonner à son goût avec de \\
\hline - $\quad$ Du sel & l'huile, la poudre des tourteaux \\
\hline - $\quad$ L'arôme & d'arachide, le sel, l'arôme, oignon et le \\
\hline - $\quad$ L’oignon & piment. \\
\hline - $\quad$ Piment & Servir \\
\hline
\end{tabular}

Dans cette recette, les graines sont préparées en association avec les feuilles de Gynandropsis gynandra, reconnues pour leur richesse en fibres, vitamines et minéraux. Les tourteaux d'arachide riches en protéines permettent d'assurer un bon équilibre en macro et micro nutriments. La source lipidique est assurée par les huiles alimentaires utilisées.

Tableau V : Recette de Lalumé de Boscia senegalensis (Bouillie à base des écorces de Boscia senegalensis)

\section{Les ingrédients :}

- Quelques branches de Boscia senegalensis

- $\quad$ Des graines de mil (Pennisetum glaucum) décortiquées

- Quelques feuilles de sabara (Guera senegalensis)

\section{Préparation :}

- Piler les branches pour enlever l'écorce

- Puis piler avec de l'eau et laisser tremper

- Vanner le mil ; une partie est lavée à l'eau et une autre partie non lavée est réservée pour la suite.

- Les feuilles de sabara sont bien préparées en prenant soin d'enlever la nervure principale et les nervures secondaires pour bien débarrasser le limbe.

- Piler les feuilles de sabara en ajoutant progressivement des grains de mil non lavé jusqu'à obtention d'une poudre.

- Ajouter l'eau des branches de Boscia senegalensis trempé pour obtenir une boule

- Piler séparément les grains de mil lavés et ajouter l'eau des branches de Boscia senegalensis

- Puis mélanger les deux boules

- Délayer avec l'eau des branches de Boscia senegalensis

- Mettre cette boule dans une certaine quantité d'eau bouillante

- Laisser cuire deux minutes et la bouillie est prête

- Servir

Au niveau de cette recette utilisée chez les femmes en post partum, ce sont plutôt les écorces de Boscia senegalensis et non les graines qui sont employées. Il est très importante de la cité parmi les recettes alimentaires, car elle est d'usage courant en milieu rural et exploite les propriétés sucrantes du 
Boscia senegalensis. La méthode de cuisson permet de sauvegarder la qualité nutritionnelle de tous les ingrédients.

Tableau VI : Recette de Graines de Boscia senegalensis plus Hassu (feuilles de Maerua crassifola)

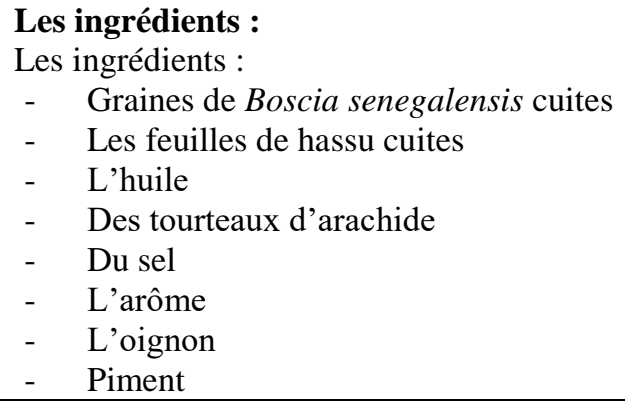

Préparation :

- Bien malaxer les graines de Boscia senegalensis seules

- $\quad$ Ajouter les feuilles et malaxer également

- Assaisonner à son goût avec de l'huile, la poudre des tourteaux d'arachide, le sel, l'arôme, oignon et le piment.

Les feuilles de Maerua crassifola sont ajoutées aux graines de Boscia senegalensis, les remarques concernant la recette 4 sont également valables à ce niveau. De façon générale la partie aérienne des plantes cumule les mêmes nutriments.

Tableau VII : Recette de Graines de Boscia senegalensis plus du lait

\begin{tabular}{|c|c|}
\hline $\begin{array}{l}\text { Les ingrédients : } \\
\text { - } \quad \text { les graines de Boscia senegalensis } \\
\text { cuites } \\
-\quad \text { du lait caillé } \\
\text { - } \quad \text { du sucre }\end{array}$ & $\begin{array}{l}\text { Préparation : } \\
\text { - } \quad \text { A l'aide d'une louche bien délayer les } \\
\text { graines de Boscia senegalensis pour } \\
\text { bien écraser les graines } \\
\text { - } \quad \text { Ajouter le lait et continuer à délayer } \\
\text { - } \quad \text { Sucrer à son goût }\end{array}$ \\
\hline
\end{tabular}

Les deux zones, objet de cette étude sont agro-pastorales, le lait est disponible surtout en saison des pluies. Cette association permet de couvrir les besoins nutritionnels des populations, le lait étant un aliment complet.

Tableau VIII : Recette de Coucous des Graines de Boscia senegalensis

\begin{tabular}{|c|c|}
\hline $\begin{array}{ll}\text { Les ingrédients : } \\
- & \text { Graines de Boscia senegalensis cuites } \\
- & \text { L'huile } \\
- & \text { Des tourteaux d'arachide } \\
- & \text { Du sel } \\
- & \text { L'arôme } \\
- & \text { L'oignon } \\
- & \text { Piment }\end{array}$ & $\begin{array}{l}\text { Préparation : } \\
\text { - } \quad \text { Bien écraser les graines de Boscia } \\
\text { senegalensis en les malaxant } \\
\text { - } \quad \text { Puis assaisonner à son goût avec de } \\
\text { l'huile, la poudre des tourteaux } \\
\text { d'arachide, le sel, l'arôme, oignon et le } \\
\text { piment. } \\
\text { - Servir }\end{array}$ \\
\hline
\end{tabular}

Ici, les graines de Boscia senegalensis sont préparées seules, puis assaisonnées avec une source lipidique (Huile), protéinique (tourteaux) et les épices. 


\section{Discussion}

$\mathrm{Au}$ Niger l'alimentation est exclusivement glucidique à base des céréales, surtout en milieu rural (FAO, 1990). Les céréales fournissent plus de $70 \%$ de l'énergie. On observe en outre que les légumineuses et les oléagineux interviennent pour $14 \%$. Les huiles végétales ne représentent que $4 \%$ des apports énergétiques de la ration alimentaire nigérienne. Parmi les groupes qui sont peu consommés, on trouve les racines et les tubercules et, de façon plus significative, les produits issus d'animaux comme la viande et les produits laitiers. La teneur en micronutriments des céréales (en particulier après mouture), des racines et des tubercules est faible, et ces aliments ne couvrent en général qu'une petite partie des besoins journaliers en la plupart des vitamines et minéraux. Dans la recherche de l'équilibre et la diversification alimentaire les populations exploitent les ressources locales disponibles, qui ne sont pas prises en charge par les statistiques agricoles et alimentaires. A l'issue de la présente étude, il ressort qu'au Niger, différents mets sont préparés à base des graines de Boscia senegalensis. Les ingrédients utilisés sont très variés et les méthodes de préparation permettent de conserver leur valeur nutritionnelle. Selon la littérature Très peu d'études ont été conduite sur les recettes. Toute fois certaines études ont fait ressortir les multiples usages des différentes parties et la valeur nutritionnelle des différentes parties du Boscia senegalensis. Des études similaires ont été menées au Burkina Faso, pays frontalier, dans une zone avec laquelle on partage le même environnement géographique. C'est ainsi que Mamounata et al. (2017) ont effectué une étude sur les connaissances ethnobotaniques de Boscia senegalensis. Les feuilles viennent en première position avec $45 \%$ des opinions suivies des fruits $(28 \%)$, des graines (19\%), des racines $(6 \%)$. Les autres utilisations relatives aux branches, au parasite (Gui) sont marginales avec $2 \%$ d'opinons exprimées. Les organes, les utilisations alimentaires ainsi que les modes d'emploi sont rapportés dans le tableau IX.

Tableau IX : contribution de Boscia senegalensis à l'alimentation des populations

\begin{tabular}{|l|l|l|}
\hline \multirow{2}{*}{ Organe } & Utilisations alimentaires & \multicolumn{1}{c|}{ Modes d'emploi } \\
\hline \multirow{2}{*}{ Fruit } & Pulpe du fruit vert consommée & $\begin{array}{l}\text { Bouillie et Mangée sous forme de petits pois, de galettes ou } \\
\text { de couscous. }\end{array}$ \\
\cline { 2 - 3 } & Pulpe du fruit mûr consommée & Mangée crue pour son goût sucré \\
\cline { 2 - 3 } & Succédanée du café & $\begin{array}{l}\text { Les graines torréfiées sont employées comme succédanée } \\
\text { du café }\end{array}$ \\
\cline { 2 - 3 } & Couscous & $\begin{array}{l}\text { Les graines séchées sont ébouillantées pour supprimer } \\
\text { l'amertume et utilisées pour la préparation du couscous }\end{array}$ \\
\hline Feuille & Couscous & $\begin{array}{l}\text { Les jeunes feuilles peuvent être utilisées pour la } \\
\text { préparation du couscous }\end{array}$ \\
\hline
\end{tabular}




\begin{tabular}{|l|l|l|}
\hline \multirow{3}{*}{ Autre } & Légumes & Les jeunes feuilles peuvent être utilisées comme légumes. \\
\cline { 2 - 3 } & Gâteaux & $\begin{array}{l}\text { Le macéré des tiges ou des branchettes donne une boisson } \\
\text { rafraîchissante au goût sucré }\end{array}$ \\
\cline { 2 - 3 } & $\begin{array}{l}\text { Le macéré des tiges ou des branchettes a un goût sucré et } \\
\text { peut ensuite servir à pétrir la pâte des gâteaux (mets } \\
\text { endogène de la région) }\end{array}$ \\
\hline
\end{tabular}

Cette étude met en évidence la contribution de Boscia senegalensis à l'alimentation des populations. Cependant, les graines sont utilisées seules, contrairement aux différentes recettes du Niger qui sont décrites dans cette étude. D'un point de vue nutritionnelle, ont rapportés que, les feuilles et les graines de Boscia senegalensis sont riches en certains acides aminés et acides gras essentiels et en certains minéraux (Kim et al, 1997 ; Julia et al, 2000). Il est donc très nécessaire que les enquêtes agricoles et alimentaires prennent en compte les aliments mal connus de façon générale et Boscia senegalensis en particulier, pour leur importante contribution à diversification et à l'équilibre alimentaire. Une ONG de la place dans la région de Zinder, Aridité Prospère "Cida Kanka » a commencé à s'intéresser aux produits et sous-produits de Boscia senegalensis (Anonyme, 2012).

\section{Conclusion}

Cette étude a concerné deux régions sur huit du Niger. Mais il s'agit des zones de grande production et de consommation des organes de Boscia senegalensis. Au-delà des multiples assertions autour de l'espèce, comme aliment de pénurie, aliment de famine, on vient de se rendre compte que Boscia senegalensis (Pers.) de son petit nom, Anza est rentré dans les habitudes alimentaires des populations. Divers mets sont préparés à base des graines, sept (7) au total répertoriés au cours de ce travail. Les ingrédients utilisés permettent d'assurer l'équilibre et la diversification alimentaire. Il revient aux statistiques nationaux, les différents acteurs qui interviennent dans l'inventaire des habitudes alimentaires et de la sécurité alimentaire et nutritionnelle, de prendre en compte l'apport de cette espèce. Aux environnementalistes de développer le réflexe de la protection et d'étudier les possibilités de culture de cette espèce, car il s'agit d'une espèce spontanée.

\section{References:}

1. Alpha Gado, B. (1989). Crises alimentaires et stratégies de subsistances en Afrique sahélienne (Mali, Burkina, Niger) aux $19^{\mathrm{e}}$ et $20^{\mathrm{e}}$ siècles [Microforme] (Doctoral dissertation). 
2. Ambouta, J. M. K., Valentin, C., \& Laverdière, M. R. (1996). Jachères et croûtes d'érosion au Sahel. Science et changements planétaires/Sécheresse, 7(4), 269-275.

3. Anonyme, (2012). Séminaire d'échanges sur les thématiques du développement du Niger, Souffles de la diaspora, N00, p.2.

4. Arbonnier, M. (2009). Arbres, arbustes et lianes des zones sèches d'Afrique de l'Ouest. Editions Quae.

5. Baumer, M. (1975). Catalogue des plantes utiles du Kordofan (Rép. du Soudan), particulièrement du point de vue pastoral. Journal d'agriculture traditionnelle et de botanique appliquée, 22(4), 81-119.

6. Baumer, M. (1981). Rôle de Boscia senegalensis (Pers.) Lam. dans l'économie rurale africaine: sa consommation par le bétail. Revue d'élevage et de médecine vétérinaire des pays tropicaux, 34(3), 325328.

7. Becker, B. (1983). The contribution of wild plants to human nutrition in the Ferlo (Northern Senegal). Agroforestry Systems, 1(3), 257-267.

8. Belem, M. O., Ouedraogo, J. Y. S., \& Nabaloum, M. (2017). Étude ethnobotanique de Boscia senegalensis (Pers.) Lam (Capparaceae) dans le Département de Banh, Province du Loroum, au Nord du Burkina Faso. Journal of Animal \&Plant Sciences, 34(1), 5390-5403.

9. Booth, F. E., \& Wickens, G. E. (1988). Non-timber uses of selected arid zone trees and shrubs in Africa (No. 19). Food \& Agriculture Org.

10. Dicko, M. H., Searle-van Leeuwen, M. J., Traore, A. S., Hilhorst, R., \& Beldman, G. (2001). Polysaccharide hydrolases from leaves of Boscia senegalensis. Applied biochemistry and biotechnology, 94(3), 225-241.

11. Doka, I. G., \& Yagi, S. M. (2009). Ethnobotanical survey of medicinal plants in west Kordofan (western Sudan). Ethnobotanical Leaflets, 2009(11), 8.

12. INS. (2012). Enquête démographique et de santé et à indicateurs multiples (EDSN-MICS IV) 2012.

13. INS. (2016). Rapport provisoir de la deuxieme enquete nationale sur les conditions de vie des menages et l'agriculture (ECVMA_2014), P.56-62

14. Kim, T. R., Pastuszyn, A., Vanderjagt, D. J., Glew, R. S., Millson, M., \& Glew, R. H. (1997). The nutritional composition of seeds from Boscia senegalensis (dilo) from the Republic of Niger. Journal of Food Composition and Analysis, 10(1), 73-81.

15. Lulekal, E., Kelbessa, E., Bekele, T., \& Yineger, H. (2008). An ethnobotanical study of medicinal plants in Mana Angetu District, southeastern Ethiopia. Journal of ethnobiology and Ethnomedicine, 4(1), 10. 
16. Maydell, H. V. (1983). Arbres et arbustes du Sahel: leurs caractéristiques et leurs utilisations. Deutsche Gesellschaft für Technische Zusammenarbeit (GTZ)/GmbH, Eschborn.

17. Mohamed Vall, A. (2009). Contribution à l'étude des plantes médicinales de Mauritanie. Ann. Univ. Lomé (Togo), 17, 9-27.

18. Musa, M. S., Abdelrasool, F. E., Elsheikh, E. A., Ahmed, L. A., Mahmoud, A. L. E., \& Yagi, S. M. (2011). Ethnobotanical study of medicinal plants in the Blue Nile State, South-eastern Sudan. Journal of Medicinal Plants Research, 5(17), 4287-4297.

19. Saadou, M. (1996), La végétation du Niger. In: L'environnement au Niger. Collection Etudes et Recherches Sahéliennes, RESADEP/PANOS. pp. 51-61.

20. Salih, O. M. ; Nour, A., \& Harper, D. B. (1991). Chemical and nutritional composition of two famine food sources used in Sudan, Mukheit (Boscia senegalensis) and Makhat (Dobera roxburghi). J-SciFood-Agric., 57(3): 367-377.

21. Valet, S. (2001). Nouvelle stratégie d'éco-développement durable par la gestion et la valorisation du report hydrique. Science et changements planétaires/Sécheresse, 11(4), 239-47. 